\title{
Intelligent Furniture Design in the Elderly Based on the Cognitive Situation
}

\author{
LU Xinhui ${ }^{1,2}, \mathrm{HE} \quad \mathrm{Li}^{1,2, \mathrm{a}}$ \\ ${ }^{1}$ Sichuan Agricultural University, 611130 Chengdu, P.R. China \\ 2 Key Laboratory of Wood Industry and Furniture Engineering of Sichuan Provincial Colleges and Universities, \\ 611130 Chengdu, P.R. China
}

\begin{abstract}
This paper analyzes the present situation of Chinese elderly furniture and the elderly has cognitive characteristics that consciousness experiences and recognitions recede, cognitive fuzzy from Information processing. Expounds the elderly intelligent furniture design elements: functional elements required the elderly furniture is easy and simple to handle; Size and shape elements should be biased towards low, light type, reduce multifunction or fold function; colour collocation should use low lightness and low purity natural materials; Emotional elements design should meet the demand of the elderly social emotion. Introduction of intelligent furniture make up the cognitive decline in the elderly, Furniture judge the elderly demand by the inductor, Supplement by hardware control module to solve the special needs of the elderly life. Build design thinking based on the cognitive process and explore the elderly intelligent furniture design. This paper discusses the design process, for example and concludes the design rules: 1.The Operating Experience Pleasure. It is the height matching of user expectation and furniture function. Pleasure in the design of the operating parts mainly embodies in two aspects. Firstly, the Fitts Law; Secondly, it's The Movement Optimization. 2."Unconscious" Design. Intelligent furniture need to delete unnecessary operation module, make it easy to understand, furniture function and cognitive scene match with each other. 3 . Modularity Design. Modularization can indirectly regulate the scale and specification of the design. Under the premise of individual character, customization, the compression of the cost, Designer should make the elderly intelligent furniture consistent with the user action.4.Design Consistency. The consistency principle reflected in the appearance, color and operation way consistency.
\end{abstract}

\section{Status quo of elderly furniture}

An investigation by The China Population and Family Planning Department showed that China social aging degree becomes more obvious in 2015. Currently, China has more than 220 million senior citizens who are above 60, more than 16 percent of the total population. China has entered the phase of aging society. With the growth of economic, the aging people purchasing power are also enhancing. Aged people pay more attention to the quality of life. But the elderly behavior speed generally slows down with age growing[1]. The body organs degradation lead to the decline of information input and output, storage, and reaction rate, the cognitive process is different from young people, so the elderly furniture design shows more particular. The elderly furniture is a serious lack in the market at present. However, Ordinary furniture can't satisfy the specific requirements of the elderly both in physiology

${ }^{\mathrm{a} C}$ Corresponding author : 490585432@qq.com Project support: 16SA0006 
and psychology, therefore, the elderly furniture $r \& d$ became a problem demanding prompt solution. For providing convenience in using furniture and ease of operation by older people, the addition of intelligent modules will solve such problems effectively.

\section{Build furniture cognitive situation of the elderly}

\subsection{The cognitive process of the elderly}

Cognitive process is a method of study human behavior and provides the basis for furniture design. Cognitive science divides people's cognitive activities into three processes: First, Cognitive mechanism which about information how to input, cognitive mechanism how to output; Second, the mental structure about cognitive how to be stored and in what form; Third, Mental Process about how to find and how to use[2].

Storage and search of memory data are the core of the whole process of information processing (Figure 1).First is the information collection stage, it is a prerequisite for memory formation, the old people's attention as information filter is hard to play normal function; Perceptual experience weakened due to the degeneration of sensory stimulation; The obstruction of information collection result in a loss of recognition.

Followed by the stage of information storage, the form of memory storage leading to confused the feel receiver in logic relationship and cognitive fuzzy between short-term memory and long-term memory due to the decline in visual and auditory sensitivity, Finally is information operation, further cognition process is derived from the sense organs interaction overlap, need a systematic approach to explain how the memory is being collected and stored.

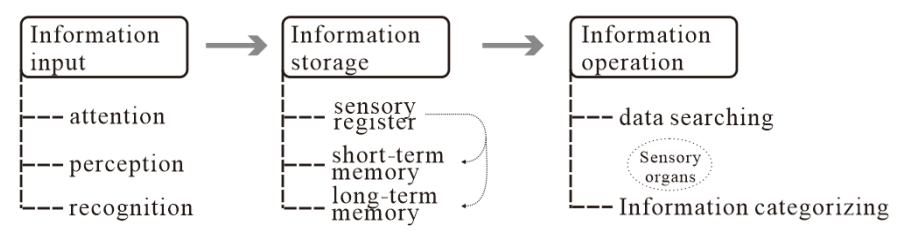

Figure1. Information processing

\subsection{The elderly furniture cognitive situation}

Furniture design considers the relationship of user, furniture and environment[3], Cognitive situation builds by interactive scenario based on the elderly psychological characterristics. In the design cognitive situation can be considered as the interactive process which the user is related to the object and the environment, that is to say, the comprehensive perception process of users towards objects and the environment. The ideal cognitive situation is to remove the boundaries between the user information handling and the use of products, which achieve the interaction coordination between the people, the object and the environment. It bring the cognition into correspondence with design elements, Intelligence make the fusion of design elements and cognition (Figure 2). The elderly furniture design elements include the function, form, colour, emotional factors and so on, as the embodiment of the furniture external characteristics. It decided by the particularity of the elderly cognitive process. The process of old people use furniture interacts with various design elements, the premise is to properly handle information. The elderly furniture use process pursues optimal emotional experience, to analyze the elderly cognitive process as a starting point, through the collection of information used to describe the object position to build cognitive situation. 


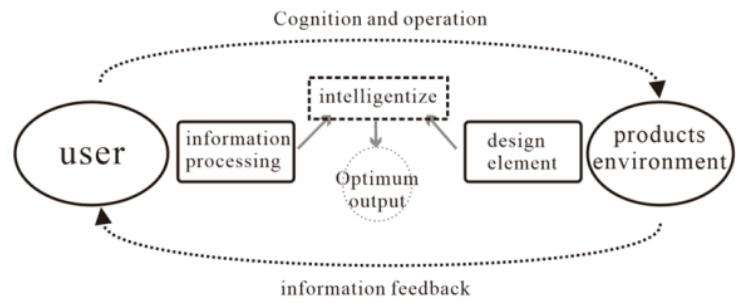

Figure2. Intelligent design and cognition process

\section{Based on cognitive scenarios of the elderly furniture design elements}

The elderly cognition is special. Based on the different characteristics of the elderly population, basic life as a starting point; this paper expounded the elderly furniture design elements from the following points:

Functional elements: Behavior is composed of a task, the task consists of an action, the action is composed of operation[4]. Hand, eye, foot as the core of the human body, best action number sequence diagram you can see from figure 3, for example, the hand operating flexibility is in turn of fingers, wrist, forearm, upper arm, shoulder, body, waist. A reasonable operation sequence can simplify the process, improve the efficiency of action. The old behavior has a slower pace; we should consider the ease of operation in the design of furniture, such as arthritis result in fingers activities inflexible. We can increase the support design, convenient old people got up and take things; The furniture should Increase the soft structure and make the corner design smooth to prevent injuring or damaging after being hit.

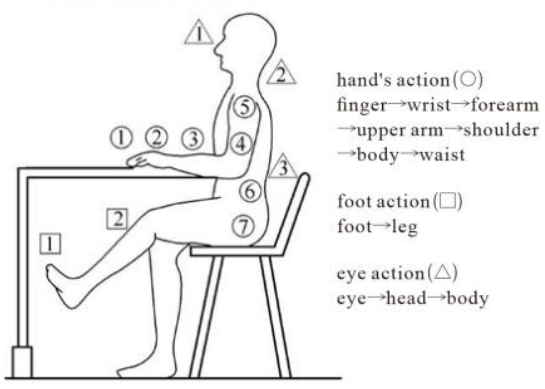

Figure3. The best action sequence of human body

Size and shape elements: The elderly furniture should comply with the old standard anthropometric data, because of the body contraction, smaller than middle-young people. Furniture should be biased towards low, light type, reduce multifunction or fold function.

Colour elements: colour collocation should match with the elderly mental feeling, with low lightness and low purity color, reducing the stimulation. Designers can choose natural texture materials to get freedom perception effect.

Emotional factors: Emotion includes three levels. They are instincts, behavior, reflect. Furniture can bring comfort, safety to the elderly, and conform to their aesthetic and meet their daily needs; are interactive on the form and function with the elderly, operate simple and interface easy to use, both entertaining; blend more emotion in the design, assist the elderly social-emotional needs, improve the superiority of life.

\section{The elderly furniture intelligent design process}


The design is a process that finds a problem and solves the problem. The reason why the elderly furniture is combined with intelligent concept is to better solve the various problems of the old life. What the elderly cognitive speed involution can make up for the intelligent design of furniture, and reduce the interaction problem of cognitive process and life scene. Design should be treated more logically and systematically other than traditional methods based on intuition and experience[5].

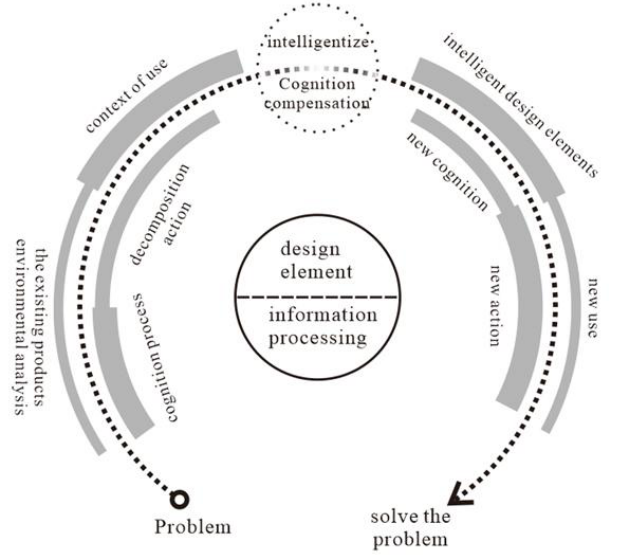

Figure4. The design process of elderly intelligent furniture

Intelligent furniture is through the sensor to collect different data information, the controller processes the information according to the preset procedures, so as to realize the automation control process of furniture. It contains two parts: hardware and software, hardware contains furniture itself, data acquisition system, control system, transmission system and other parts, The software includes data acquisition program and control program. Based on the cognitive process of intelligent furniture design, the core is a systemic treatment of product design elements, make design elements and cognitive processes (i.e., information processing) seamless integration (figure 4). The following is the specific design process.

\subsection{Raise a question}

Problem is divided into two levels: In terms of the elderly itself, their cognitive speed, movement speed slow down, the body best action position have changed depending on the specific environment; In terms of the furniture and the environment, the traditional furniture, argued that classification, form, function, size should aim at the older users demand by the functional partition, amounting to little more than a traditional space division, leading to not match the cognitive process and furniture design elements in older people.

\subsection{Build usage scenarios}

Interaction is the center in usage scenarios, classified according to the functional characteristics. Idealized cognitive situation is that cognitive exactly match with furniture operation function in the elderly, It is the unity of the cognitive processes and design elements. Building the old furniture usage scenarios should be accurate to specific groups and space environments, such as the bedside table is a assist function at bedtime which can put on cups, desk lamps, drugs, glasses, etc. The usage scenarios is sitting, lying in bed, when designing in addition to action factors, such as Table layout, air motion, temperature and humidity should be considered. The auxiliary furniture should reduce operating action.

\subsection{The addition of intelligent elements}


Intelligent elements are the key to realizing intelligent furniture. Intelligent furniture realizes by two parts, hardware and software. The feasibility analysis includes the information operation and user demand matching in the software part. In the hardware part it requests that the information collections, transmission parts, furniture are consistent with user operations. How to maximize identify of needs and feedbacks. Taking an example, the elderly watching TV on the sofa, they need a rest after sitting for a long time. Intelligent sofa collects information and need to make a corresponding mechanical adjustment when nodding off. Such as changing to the horizontal state, and regulating temperature for conforming rest. The sofa has low seat height. It can elevate the seat surface when old people get up, etc.

\subsection{New design whether meets the usage scenarios}

Whether the cognitive process matches with design elements, whether the user uses fewer moves to finish the task than before, it is the criterion of intelligent elements implant success and assessment phase of the whole design process.

\section{The elderly intelligent furniture design principle}

Regarding the design of intelligent furniture in elderly, on behalf of the user's demand cognitive situation is implicit. Design elements are dominant and are the function of furniture. Elderly intelligent furniture design on the cognitive scene construction as the breakthrough point, consider the design elements to what form to reflect the user's potential demand. In what ways did the elderly communicate with furniture, is the furniture design principles.

\subsection{The Operating Experience Pleasure}

Pleasure is the height matching of user expectation and furniture function. Whether the furniture function meets the user predict demand can provide the excellent feedback for the furniture. Operation experience makes the product operation interface consistent extensively with the user's cognitive process based on the user's cognitive situation. In the process of the interaction of the interface with intelligent furniture in the elderly, intelligent furniture should bring all the necessary information and tools to users. The furniture multi perceive specific state by sensors and make the user operation of the furniture in the controllable range. The user should be independent in a variety way of perception in interactive. If the intelligent control of the products conflict with the user's demand, should give in to the user.

Pleasure in the design of the operating parts mainly embodies in two aspects. Firstly, the Fitts Law. That is to say when the user is in a particular use state, the operating objects size, distance should be ease to use. Secondly, it's The Movement Optimization. By changing the operation action or improving operations position and the use object height is the action to reduce operation, make more convenient and effective.

\section{2 "Unconscious" design}

Ideally, product is no learning process, make users feel easy to use for the first time. In fact, there is no such thing as absolute without the learning process of operation, so you need to consider the learnability and availability of intelligent furniture. Elderly intelligent furniture must consider using frequency firstly, should make the higher of use, the more simple.

"Unconscious" is the process that objects drive the information storage to achieve operation by a certain characteristic representation. Intelligent furniture on man-machine interface processing should activate the old memories of the experience and associate with the real world in the design. For 
example in an easier way to arouse the user's cognition, control interface should use common symbols, software algorithm match with the cognitive process, simplify all unnecessary features.

The "unconscious" Operating process is the interactive object to be understood easy and the embodiment of interface humanization. Product interface is the medium of transmission and the exchange of information between people and machine. Is also a media of communication between them[6]. The old memory degradation, intelligent furniture need to delete unnecessary operation module, make it easy to understand, furniture function and cognitive scene match with each other.

\subsection{Modularity Design}

The elderly intelligent furniture can be divided into two categories, software module and hardware modules. Although the functions of furniture make a difference in different usage scenarios, Old people as a kind of user groups, it drives the elderly furniture homogeneity on the intelligent control based on the cognitive process and the usability operating principle.

In the aspect of hardware, its modular mainly reflects on the control plate and the transmission structure. Modular hardware is initial form portion of the intelligent furniture, can adapt to the user's personality needs and customization, the modular control unit has strong learnability, easy to form a good user experience. The transmission structure modularization can indirectly regulate the scale and specification of the design. Under the premise of individual character, customization, the compression of the cost, Designer should make the elderly intelligent furniture consistent with the user action.

\subsection{Design Consistency}

The consistency of the intelligent furniture reflected in how to correctly guide the user operation reliability, and able to comply with the laws of formal beauty. The consistency principle reflected in the appearance, color and operation way consistency. Appearance consistency not only does In line with the aesthetic demand from the perspective of formal beauty but also makes product seriation. Color consistency is mainly aimed to distinguish an operation module with the furniture in the elderly intelligent household and give consideration of aesthetic factors. Operation consistency includes hardware drive and software control. Interact has standard specifications, needs to keep consistent and stable to offset the elderly exclusive, for new things to improve their cognitive acceptance.

\section{Conclusions}

Intelligent furniture is the main way of solving the communication of cognitive process and design elements. The elderly cognitive speed slowed down, need to provide a positive response in the intelligent furniture based on cognitive situation. Furniture judge the elderly demand by the inductor, Supplement by hardware control module to solve the special needs of the elderly life. Build design thinking based on the cognitive process and explore the elderly intelligent furniture design.

\section{References}

1. Hale S, Lima SD, Myerson J, Paychology and aging, 6,512 ( 1991)

2. Chiu-Shui Chan, Design Cognition_Cognitive Science in Design,15(2008)

3. LU Xin-hui , ICSREI,5,(2014)

4. Reimann R, Cronin D, Cooper A, Electronic Industry Press,(2012)

5. Chiu-Shui Chan, Design Cognition_Cognitive Science in Design,29(2008)

6. XU Shi-hu, LU Xin-hui, The packaging engineering,33,64(2012) 